\title{
Rapid Extraction of Target Information in Messy Multimedia Medical Data
}

\author{
Mai Xin'1, Zhifeng Ye1, Changhua Chen1', Yuhan Cai' ${ }^{2}$, Haili Ding1, Ran Wang1, Shiqian Wang1 \\ ${ }^{1}$ Library, Nanjing University of Aeronautics and Astronautics, Nanjing, China \\ ${ }^{2}$ Sichuan Hangbiao Electric Power Construction Co., Ltd., Meishan, China \\ Email: jiangwei_xm@aliyun.com
}

How to cite this paper: Xin, M., Ye, Z.F., Chen, C.H., Cai, Y.H., Ding, H.L., Wang, R. and Wang, S.Q. (2019) Rapid Extraction of Target Information in Messy Multimedia Medical Data. Intelligent Information Management, 11, 38-41.

https://doi.org/10.4236/iim.2019.112004

Received: February 24, 2019

Accepted: March 17, 2019

Published: March 20, 2019

Copyright (C) 2019 by author(s) and Scientific Research Publishing Inc. This work is licensed under the Creative Commons Attribution International License (CC BY 4.0). http://creativecommons.org/licenses/by/4.0/

\begin{abstract}
The information work in the hospital is very complicated. The amount of data is huge, and the forms are various. It is very troublesome to organize and find the data you need. In order to solve the problem, which often appears in the process of analyzing medical case and data, we use Labview2017 to design the corresponding multimedia data calling system for the purpose of rapid searching and effective extraction. This program, which greatly improves work efficiency, can realize fast searching multimedia data in the folder. Since we use of this type of system, the work efficiency has been greatly improved, and the burden on the staff has been greatly reduced.
\end{abstract}

\section{Keywords}

Multimedia, Information Retrieval, LabVIEW2017, Fast Searching

\section{Introduction}

With the advancement of times, people's requirements for office efficiency are getting higher and higher [1], which makes the level of office automation systems correspondingly higher [2].

In the daily information statistics of large hospitals, there are often huge folders of messy storage. It is very troublesome to organize such folders and obtain specific information in need [3]. When the data are stored in multimedia style, the file retrieval function of the operating system alone is difficult to meet the demand [4] [5] [6]. Using the search system that comes with the computer system, you can only retrieve files simply with inefficient [7]. If the corresponding data is damaged or cannot be used, it requires manual discrimination.

Designing a kind of software which can quickly retrieve and access the contents of multimedia files to improve the retrieval efficiency before manual dis- 
crimination is very necessary in office practice.

Through further research work, we fully understood the needs of our staff. After that, work progress has been made and each task node has been controlled. On the basis of active communication and collaboration among all personnel, we conscientiously implemented relevant work.

\section{Construction of This System}

The process is shown in Figure 1: By setting the retrieved folder as a file path, we use the software written to do the following work. The files in the folder are matched according to the requirements of the retrieved fields, and then the files in various formats containing the retrieved fields are called for review.

After that, they are directly opened by the default matching software for the operator to filter and decide the trade-off. It saves the manual verification process, which needs to open one by one. Using this method, we greatly improve the work efficiency.

Considering that the designed system should have the requirements of cross-platform, simplicity and rapidity, Labview2017 with good portability and operability is used as the development platform to design the software system. The designed operation interface and program interface are shown in Figure 2 and Figure 3.

\section{Experimental Argumentation}

The following is a demonstration by experiment. The searched folder is input in accordance with the search path shown in Figure 2, and the types of files corresponding to "Type 2" in the folder shown in Figure 4 which are matched and searched. We do it in this way to determine which information about type 2 diabetes is needed and which is useless, then get useful information and organize messy folders.

The results of various formats, which meet the search conditions, are all listed quickly and opened by their default software for the searcher to view, as shown in Figure 5.

\section{Conclusions}

It can be seen from the results shown in Figure 5 that the system can smoothly achieve the desired target, and also can quickly call data in various formats to realize the function of searching and retrieving data.

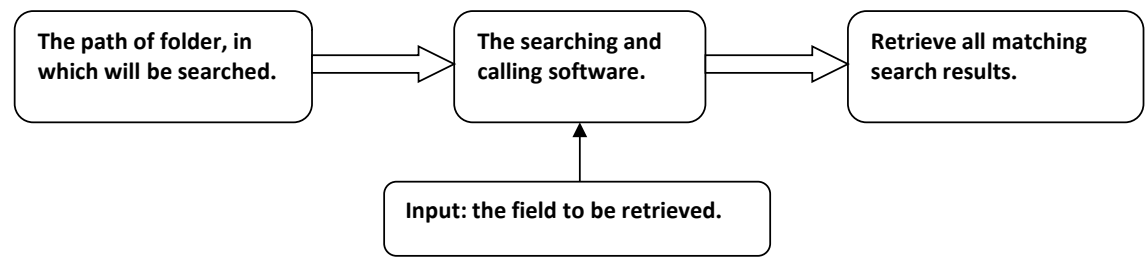

Figure 1. The process of system. 


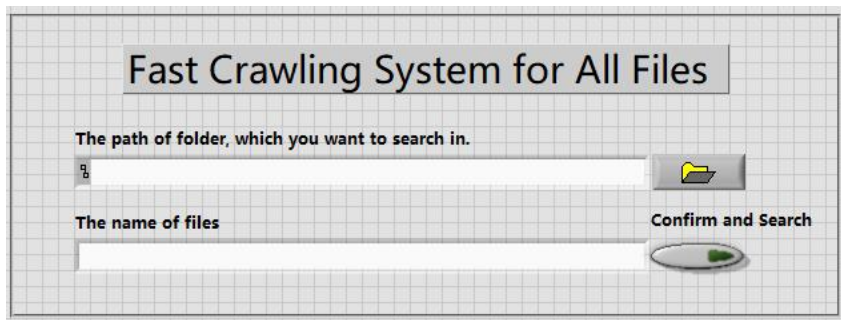

Figure 2. System operation interface.

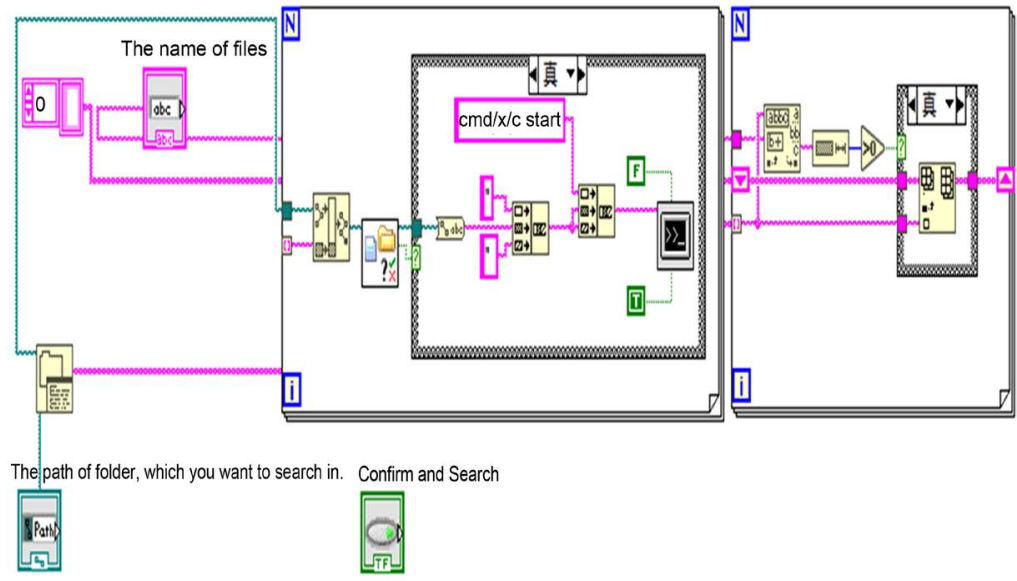

Figure 3. System program interface.

\begin{tabular}{|c|c|c|}
\hline 国|Anisodamine & 띰Anisodamine & 䛗 Anisodamine \\
\hline 滒 Anticonvulsant & 띰Anticonvulsant & 剾 Anticonvulsant \\
\hline 国Antioxidant stress & Antioxidant stress & Antioxidant stress \\
\hline 涪 Antioxidant stress & 띰Antioxidant stress & 啮 cataract \\
\hline 삠 cataract & 国 cataract & 딱 Cerebrovascular disease \\
\hline 띰Cerebrovascular disease & Diabetic cardiovascular complications & Diabetic cardiovascular complications \\
\hline Diabetic cardiovascular complications & 诃Diabetic cataract & 밈 Diabetic cataract \\
\hline Diabetic cataract & Wiabetic cerebrovascular disease & ⿹ㅣㄹ Diabetic cerebrovascular disease \\
\hline 區 Diabetic cerebrovascular disease & 欧Diabetic foot & 띰 Diabetic foot \\
\hline Diabetic foot & 卧 Diabetic ketoacidosis & 犒Diabetic nephropathy \\
\hline 삠Diabetic nephropathy & 国 Diabetic nephropathy & 国Diabetic neuropathy \\
\hline 띰 Diabetic neuropathy & 區 Diabetic neuropathy & Diabetic retinopathy \\
\hline 昜Diabetic retinopathy & 澏 Diabetic retinopathy & 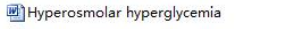 \\
\hline 띰 Hyperosmolar hyperglycemia & isit Hyperosmolar hyperglycemia & 回 Ketoacidosis \\
\hline 可 Ketoacidosis & 뜸 Ketoacidosis & 䶅 Ketoacidosis \\
\hline 凅Ketoacidosis & 팜 Lactic acidosis & 띰 Lactic acidosis \\
\hline Lactic acidosis & 삘 Neurotrophic factor & 띰 Neurotrophic factor \\
\hline [it Neurotrophic factor & 맘 Retinopathy & 冈n Retinopathy \\
\hline 㗱 Retinopathy & [2] Type 2 diabetes complications & 땜 Type 2 diabetes patient \\
\hline 北Type 2 diabetes treatment case & 띰 Type 2 diabetes treatment case & (涵Type 2 diabetes treatment \\
\hline 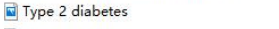 & 击Type 2 diabetes & 띰 Type 2 diabetes \\
\hline 醐 Type 2 diabetes & 凅Type 2 diabetes & 北Uveitis \\
\hline
\end{tabular}

Figure 4. Cluttered folder to be retrieved.

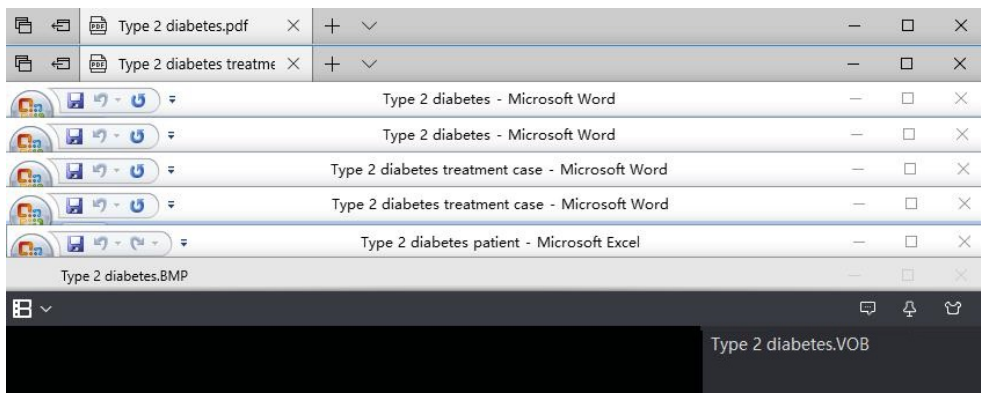

Figure 5. System operation result. 
Thereby, the management efficiency of the file data is greatly improved, and the consumption of the operator's energy in the data sorting and data management is effectively reduced.

\section{Acknowledgements}

At that period, we nearly spent all our time in hospital. We did a lot of work in this field. At last, we achieve our goal and get progress. Thanks to all ones of our team, we finish this work successfully.

\section{Conflicts of Interest}

The authors declare no conflicts of interest regarding the publication of this paper.

\section{References}

[1] Dan, M.I. (2018) Application of Office Automation in the Management of Documents and Archives. China Health Standard Management, 13, 6-8.

[2] Wang, J., Chen, J.-L., Li, Z.-J. and Su, Y. (2018) Discussion on Management of Hospital Commercial Contract Based on Office Automation. Hospital Management Forum, 8, 73-74.

[3] Fu, T. and Quan, Y. (2018) Paperless Hospital Management. Modern Hospital Management, 4, 82-84.

[4] Tan, C. (2018) The Construction Research on OA Office System by Changsha Construction Investment \& Development. Hunan University, Changsha.

[5] Sun, Y.F. (2018) Design and Implementation of Office Automation System Based on SOA. Tianjin University, Tianjin.

[6] Srivastava, H.S., Sivasankar, T. and Patel, P. (2018) An Insight into the Volume Component Generated from Risat-1 Hybrid Polarimetric Sar Data for Crop Biophysical Parameters Retrieval. ISPRS Annals of Photogrammetry, Remote Sensing and Spatial Information Sciences, $I V-5,5,209-214$.

[7] Ye, N., Walker, J.P., Rüdiger, C., Ryu, D. and Gurney, R.J. (2018) Remote Sensing; Investigators from Monash University Have Reported New Data on Remote Sensing (Surface Rock Effects on Soil Moisture Retrieval from L-Band Passive Microwave Observations). Journal of Technology \& Science, 10, 33-43. 